\title{
Acute Pneumonia: it is Interesting to know Who and When Refuted and Abolished the Biological Laws of Inflammation? (Actual Questions to Specialists)
}

\author{
Igor Klepikov* \\ Pediatric surgeon, Renton, USA \\ *Corresponding author: Igor Klepikov, Pediatric surgeon, Renton, USA
}

\begin{tabular}{|c|c|}
\hline ARTICLE INFO & ABSTRACT \\
\hline Received: 幽 December 13, 2019 & Citation: Igor Klepikov. Acute Pneumonia: it is Interesting to know Who and When Re- \\
\hline Published: 彗January 06, 2020 & $\begin{array}{l}\text { futed and Abolished the Biological Laws of Inflammation? (Actual Questions to Special- } \\
\text { ists). Biomed J Sci \& Tech Res 24(2)-2020. BJSTR. MS.ID.004011. }\end{array}$ \\
\hline
\end{tabular}

\section{Opinion}

Acute Pneumonia (AP) over the past decades, constantly occupies a place in the list of nosologies, among the leading causes of morbidity and mortality. The same stability and perseverance characterize efforts to address this problem. The fixation of attention on the pathogens of AP and the main emphasis on their suppression for many years have been presented as the main guarantee of successful treatment of the disease. If these efforts, based on this view, were confirmed by encouraging results, there would be no point in discussing the problem. Unfortunately, the results of AP treatment tend to be the opposite of the desired and predictable. The gradual decline in the effectiveness of antibiotics, the emergence and continued growth of antibiotic-resistant strains, the increase in the number of patients with various complications of the disease have long required finding ways out of this situation. The discovery of antibiotics is one of the outstanding achievements of medical science of the $20^{\text {th }}$ century, and the triumph of their initial application has raised great hopes for the future. But over the years, it has become increasingly clear that the new dream of a universal cure for many diseases is fading into history, and the growing implications of this endeavor are raising more and more questions.

In the gradual process of changing the conditions and outcomes of antibiotic use, one would expect to find other solutions. However, contrary to the logic of the way out of this situation, the narrow direction of antimicrobial action has become an almost dominant reflection of modern AP doctrine. Moreover, the disease, which has never in its history been classified as contagious, is now considered infectious. The current generation of doctors is brought up on the exaggerated role of antibiotics in the treatment of such patients. Therefore, the change of views on the nature of AP and shifting the focus to choosing the right therapeutic efforts can present great challenges for current professionals in overcoming existing stereotypes. Another thing is surprising: these stereotypes of views have been formed for years with the immutable inviolability of the fundamental provisions of medical science and, of course, such basics of medical knowledge should be familiar to graduates of medical universities. In order to understand the essence of modern misconceptions about the nature of AP, the easiest way to try to refute the following statements. If such a refutation can be argued and proved, then other solutions should be sought, and if not...?

1. AP regardless of the introduction of new terms (community-acquired, healthcare-associated, hospitalacquired, ventilator-associated etc.) remains a classic example of acute inflammation of nonspecific etiology.

2. If the basis of this disease is an inflammatory process, then it should be inherent in the following significant signs.

A. Inflammatory transformation of tissues occurs due to the vascular reaction in the lesion, which is accompanied by a change in the permeability of vascular membranes and strictly sequential change of stages. 
B. The rate of development of inflammatory transformation is determined by the ability of the body to respond to the emerging focus of irritation and is an individual characteristic that can have both a lightning nature and a relatively smooth development.

C. In the most aggressive cases, the inflammatory process can proceed at lightning speed by the type of Artus reaction. In such situations, it is possible to develop necrosis of inflamed tissues within a short time, and the body has great difficulty adapting to sudden functional disorders.

D. Like any other inflammation, AP is accompanied by five classic signs, which after their description by Celsus and Galen for several centuries are a mandatory characteristic of such diseases (heat, pain, redness, swelling, and loss of function).

E. Of the five above-mentioned signs, the most practical importance is the loss of function, which, depending on the localization of inflammation, determines the clinical features and severity of the disease.

3. Of all the currently known list of acute nonspecific inflammatory processes, AP is the only one that develops in the vessels of the small, not the large circle of blood circulation.

4. One of the important functions of the lungs is their integral regulatory role in the circulatory system of the body. Through the vessels of the pulmonary circulation passes all the blood circulating in the body and for the normal synchronous operation of the two halves of the heart, the blood pressure of the large circle should be several times higher than this indicator in the pulmonary vessels. Acute inflammation in the lung tissue affects the receptors of blood flow regulation in the small circle of blood circulation, including mechanisms of unloading of pulmonary vessels with a tendency to reduce systemic blood pressure.

5. Antibiotics have only antimicrobial action and are not able to directly affect the mechanisms of the inflammatory process. Here were pointed out only the most important, from my point of view, axioms of medical science, which are still indisputable and can facilitate the understanding of the reasons for the low efficiency of modern treatment of AP.

Therefore, if we continue to consider the same antibiotic as the main means of treating completely different diseases, the list of which includes pneumonia, what success can be expected with such a distribution of one drug among different categories of patients? When "antibiotics alone" do not give the desired result, additional methods of General therapeutic nature are included, the effect of which in AP is not objectively evaluated. Even such a method of assistance in AP, as oxygen therapy, which is of the most specific nature and is used allegedly because of the violation of gas exchange in the area of inflammation, raises a reasonable question:
"Why a small focus of acute inflammation in the lung causes more severe hypoxemia than atelectasis of the lobe or even the entire lung?" It is possible to significantly expand the understanding of the pathogenesis of AP and radically change the results of treatment of these patients only by revising the doctrine of the disease. This first step in solving this problem is not only difficult because of the need to break the stereotypes that have developed over the years, but also necessary to achieve real, not declarative, success. The first step in this direction was taken more than 30 years ago. Clinical testing of other approaches to the treatment of AP, arising from new ideas about the nature of the disease, confirmed the validity of the chosen course.

This work was performed and tested in clinical conditions in 1976-1985 in the clinic of pediatric surgery of the state Institute of advanced training (Novokuznetsk, USSR). Unfortunately, life circumstances did not allow the author to continue the research, to develop the results achieved and to present them at the international level in a timely manner. Over the past period, there have been many significant events, in particular, instead of the collapsed Soviet Union, another country appeared on the map. The above-mentioned institution in which the author worked no longer exists, having radically changed its status. However, the solution of the AP problem remained true to the previous direction and only accumulated new questions and tasks. In this regard, research and clinical trials of pathogenetic approaches to the treatment of patients with AP and the results of this work can provide answers to a number of questions facing us today, which allows us to consider the research of thirty years ago relevant and worthy of mention. The peculiarity of this material was that the most severe patients with initial forms of AP were concentrated in the surgical Department. Such hospitalization of non-surgical patients was explained by the rapid development of complications, high mortality and lack of conditions for intensive care and resuscitation in other hospitals.

The administrative decision regarding the concentration of severe patients did not correct the situation and did not change the results of treatment. At that time, the aggressiveness of AP development was explained solely by the presence of Staphylococcus in the development of the disease, although some facts contradicted this opinion. That is why the search for a solution to the problem was started with a revision of views on the nature of AP. The main and first step in the study was a radical revision of views on the nature and mechanisms of development of AP. The new doctrine was based on well-known scientific medical axioms and facts that already had the previous justification and confirmation. In addition, the following studies were carried out:

a) Experimental model AP (4 series of experiments, 44 animals) to obtain a model of pleural complications (certificate for invention № 1631574, A1, November 1,1990, USSR).

b) X-ray examination with contrast of 56 anatomical lung 
preparations with various forms of AP taken from deceased patients.

c) Record of comparative rheopulmonography before and after treatment procedures (36 patients).

d) Analysis of monitoring and treatment of 994 children with AP and its various destructive and pleural complications [1].

The revised treatment guidelines were applied in 101 patients in the initial period of aggressive forms of AP. The analysis of the results showed that compared with the same group of patients who received the previous complex of care, the number of pleural and pulmonary complications significantly decreased $(\mathrm{T}=8,65 ; \mathrm{P}$ $<0,001)$, the length of hospital stay was reduced by three times, respectively, material and financial costs of treatment were significantly reduced, there were no deaths. The received results allow to speak about possibility of the guaranteed prevention of suppurative and destructive complications of the disease. The revised treatment package was also used in 102 patients who already had pleural changes at the time of hospitalization, despite the initial period of the disease. In this group of patients, rapid recovery without fatal outcomes was also achieved, but the results depended on the nature of the initial pleural reaction.

The more intense and saturated the pleural effusion was at the time of hospitalization, the greater was the probability of the appearance of destructive cavities in the lung with the resolution of inflammatory infiltration. The main results of research, clinical trials and the results obtained can be summarized as follows. AP is a classic inflammatory process of nonspecific etiology, where the microbial factor may be one of the trigger causes, but not its main source. In the presence of appropriate conditions, many microorganisms present in the body as a concomitant microflora can take part in the inflammatory process. At the same time, the true participant of this process in the vast majority of patients remains unknown, and discussions about the etiology of the disease, as a rule, are based on indirect studies, which cannot be considered an objective argument and do not have a significant impact on the final results [2,3]. However, in patients with relatively slow development of the disease, treatment by the type of "antibiotics alone" manages to suppress the revolt of the microflora and enable the body to cope with the problem. Despite the significant decrease in the effectiveness of antibiotics compared to their first results, such observations support the illusion of the exclusive role of these drugs, as well as the impression of the infectious nature of AP.

Therefore, when this type of treatment does not give the desired result or the disease initially begins aggressively and requires the use of additional methods of care, the situation is out of control of the attending doctors. It is in the latter case that it is very important to revise the therapeutic principles and to change the emphasis in the list of efforts. Antibiotics in AP, based on my previous experience, are an important but supportive treatment for this category of such patients. Their choice should be determined empirically (this choice continues to prevail today), considering the possible participation of different variants of microflora. It is necessary to exclude intravenous infusions, which belong to the inevitable methods of care for every hospitalized patient, but which have a stimulating effect on the inflammatory transformation in the lung tissue [4]. The first aid to patients with AP should include methods of influence on pathogenetic mechanisms of inflammatory process in lungs and have objective, instead of illusory confirmation of its positive effect [5]. Further intensive restoration of bronchial patency and reaeration of the lungs should maintain and develop resistance against the included dynamics of inflammation. The possibility of using other approaches to the treatment of AP will not even seem a real prospect without first radical revision of views on the nature of this disease. Therefore, the revision and modification of the AP doctrine is a necessary and inevitable step in this direction [6-9].

\section{References}

1. Klepikov I (1989) Acute pneumonia and its purulent and destructive complications in children in the midst of a major industrial center of Western Siberia. Dissertation for the degree of Doctor of Medical Science, Leningrad, 1989.

2. I Klepikov (2018) Acute Pneumonia: What Infection We're Treating? Res Pediatr Neonatol 3(1): 014.

3. Klepikov Igor (2019) Associations and reality in the etiology of acute pneumonia. J Clin Rev Case Rep 4(2): 1-5.

4. Igor Klepikov(2017) The Effect of Intravenous Infusion on the Dynamics of Acute Pneumonia. EC Pulmonology and Respiratory Medicine 4(1): 15-20.

5. Igor Klepikov(2018) First Aid for Aggressive Forms of Acute Pneumonia. EC Pulmonology and Respiratory Medicine 7(2): 34-37.

6. I Klepikov (2019) Acute Pneumonia: Biological Rules and Laws require Attention and Respect. Journal of Respiratory Diseases 1(1): 25-29.

7. Klepikov I (2017) The new doctrine is the future solution to the problems of acute pneumonia. Gen Med Open 1(3): 2.

8. Klepikov I (2017) Acute Pneumonia in Children - Illness or Infection? Open Letter to the Editorial Staff. J Infect Non-Infect Dis 3: 022.

9. Igor Klepikov (2019) Mortality in Acute Pneumonia: Fatal Inevitlitabiy? 


\section{ISSN: 2574-1241}

DOI: 10.26717/BJSTR.2020.24.004011

Igor Klepikov. Biomed J Sci \& Tech Res

(C) This work is licensed under Creative

Submission Link: https://biomedres.us/submit-manuscript.php

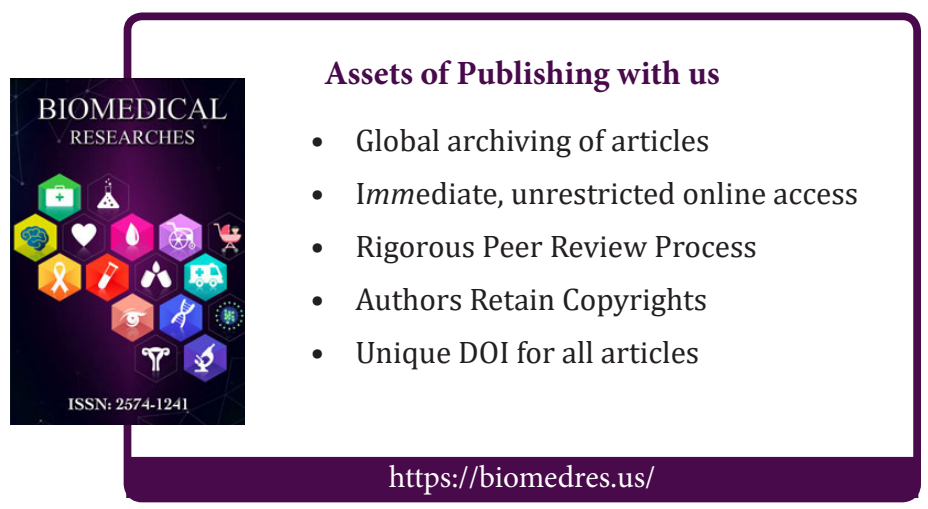

